\title{
Novel ROADM modelling with WSS and OBS to Improve Routing Performance in Optical Network
}

\author{
Kavitha G.R.*, Indumathi T.S.** \\ * Dept. of Telecommunication Engineering, Dr. Ambedkar Institute of Technology, Bangalore, India \\ ** Dept. of Digital Electronics and Communication System, V.I.A.T, V.T.U Muddena Halli, Chickballapur District,
} India

\begin{tabular}{l} 
Article Info \\
\hline Article history: \\
Received Jun 8, 2015 \\
Revised Dec 7, 2015 \\
Accepted Dec 29, 2015 \\
\hline Keyword: \\
Blocking Performance \\
OBS \\
Optical Network \\
ROADM \\
WSS
\end{tabular}

\begin{abstract}
The area of optical network is on constant point of focus among the research communities owing to potential advantage of long distance communication as well as the routing issues associated with it. Majority of the implemented techniques calls for using expensive hardware or adopting narrowed technical assumptions, for which reason the routing performance couldn't be optimized to a large extent. The present manuscript proposes a novel technique called as Optimized Routing for Peak Traffic under the scenario of uncertainties in the existing traffic scenario. The proposed solution presents some potential principle that is incorporated in the ROADM design for enhancing the capabilities of the proposed system. The system is evaluated under multiple scenarios of analysis using blocking probabilities, OSNR, bit error rate, iteration etc. The outcome of the proposed system is compared with the one of the existing significant study to benchmark it.
\end{abstract}

Copyright (C) 2016 Institute of Advanced Engineering and Science. All rights reserved.

\section{Corresponding Author:}

Kavitha G.R.,

Department of Telecommunication Engineering,

Dr. Ambedkar Institute of Technology

Bangalore, India,

Email: kavigr@gmail.com

\section{INTRODUCTION}

Optical network is the area of communication that deploys the transmitted signal in the form of light among the various nodes in the telecommunication network [1]. This is the most upcoming form of the telecommunication network that will finds its higher technical adopt in near future [2]. With the massive growth of the requirement of channel capacity with the growing customer, Wavelength Division Multiplexing (WDM) technology is being frequently adopted to cross-counter this challenge. The optical network is required to be highly flexible, sustainable, as well as controllable [3]. Hence, majority of the research attempts are in the direction of mitigating the issues of optical network pertaining to make the networking system more versatile. One of the important and highly demanding factors of the optical network is to posses the capability to instantiate and provides bettered connectivity with higher dimensionality of the network sustainance. Network interoperability as well as scalability is another significant issue in optical network, which are yet to be seen with standard solution in research community. In the design of optical network, reconfigurable optical add or drop multiplexer (ROADM) plays a crucial role. ROADM (also represented by node) is basically an optical sub-system that area capable of selecting (adding) or removing of a particular wavelength present in an optical cable [4]. Some of the significant charecteristics of the ROADM model are i) spontaneously monitors power of the signal, ii) performs equalization of power, and iii) higher degree of supportability of all data rates, modulation formats, as well as protocols. This paper discusses about a novel approach that uses the refined version of the ROADM model. The evaluation of the study has been performed using WSS and OBS. The proposed study has considered some of the critical constraints and 
consideration for ensuring that proposed study can ensure peak traffic consideration. The discussion of introduction in Section 1 is followed by Section 1.1 that highlights about background of study followed by problem discussion in Section 1.2. Section 1.3 introduces the proposed model followed by elaborated discussion of research methodology in Section 2. Section 3 discusses about the Result and discussion being accomplished from the study along with performance comparative analysis that is further followed by conclusion in Section 4.

\subsection{Background}

There are substantial amount of literatures that has been introduced in the last decade for enhancing the network performance of optical network. Chau et al. [5] have presented a mathematical model for hierarchical optical path networks focusing on problem of waveband add/drop ratio constraint. Gianluigi et al. [6] have used network global expectation model for enhancing scalability analysis of WSS based ROADMs. Roy et al. [7] have used Digital Grooming for Analyzing the Multilayer switching. Karamitsos et al. [8] have used LMS algorithm for Resource Reservation Mechanism for OBS. Usage of Mach-Zehnder interferometers was seen in work of Miller et al. [9]. Luo et al. [10] have used Markov chain for the distribution focusing on issues of Blocking Probability of Multifiber WDM optical networks. Gangaya et al. [11] have used Bernoulli and Poisson Splitting Model for enhancing Blocking probability based on the Network and Physical Layer. Chu et al. [12] have used i) Passive rerouting with full wavelength and ii) Passive rerouting without wavelength for improving the blocking performance in Lightpath reroutingin optical WDM networks. Bahleda et al. [13] have used Barry and Humblet for enhancing Blocking probability. Ansari et al. [14] have used random wavelength assignment and first fit algorithm while J.Jeno Jasmine et al. [15] have used feedback routing algorithm. Zhang et al. [16] have used Dijkstra's algorithm to study the problem of Semi-lightpath in a wavelength routed optical networks. Lu et al. [17] have used DIR and SIR for studying blocking probability. However, closer looks into the work are found with following issues i.e. i) Less consideration of the real-time constraints in optical network, ii) Relying on conventional ROADM with significant issues of optimization still unaddressed, iii) Usage of expensive hardware or proprietary tools posing a natural limitation of narrowed evaluation, iv) No consideration of real-time uncertainty in traffic behaviour been even considered or attempted to model, v) No significant or extended modelling technique to ensure optimal routing in peak traffic condition, vi) Traffic based modelling based on OSNR, ROADM, WSS, and probability theory was never attempted before. Hence, the proposed system have identified these research gaps and proposed a model for addressing the same.

\subsection{Problem Identification}

The identified problems of the study are:

- $\quad$ Less consideration of the real-time constraints in optical network.

- Relying on conventional ROADM with significant issues of optimization still unaddressed.

- Usage of expensive hardware or proprietary tools posing a natural limitation of narrowed evaluation.

- No consideration of real-time uncertainty in traffic behaviour been even considered or attempted to model.

- No significant or extended modelling technique to ensure optimal routing in peak traffic condition.

- Traffic based modelling based on OSNR, ROADM, WSS, and probability theory was never attempted before.

\subsection{Proposed Solution}

Our initial study [18] has reviewed various scientific approaches discussed by prior authors. The mathematical approach of our prior ECON model [19] has proved that it is possible to re-define ROADM and enhance the throughput of the networking system involved in ROADM. However, the study carried out by Pan et al. [20] doesn't have any consideration of ROADM module, which was the main distinction factor with ECON model that has used a simplistic design of ROADM to enhance the OSNR. It was more important that design of ROADM module to be tested more effectively using more standard technical parameters for understanding the optimal performances of optical network. The architecture of the propsoed system is as shown in Figure 1 


\begin{tabular}{|c|c|c|c|c|c|}
\hline \multicolumn{6}{|c|}{ Optical network Parameters } \\
\hline \multirow{5}{*}{ 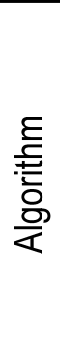 } & Enhance & nent Principle & \multirow{2}{*}{\multicolumn{2}{|c|}{$\begin{array}{c}\text { Wavelength } \\
\text { Selective } \\
\text { Switching }\end{array}$}} & \multirow{5}{*}{ 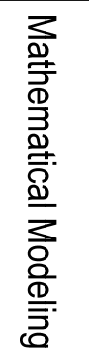 } \\
\hline & Propo & ed ROADM & & & \\
\hline & \multicolumn{4}{|c|}{ Queuing network Model } & \\
\hline & \multicolumn{4}{|c|}{ Continuous Time MARKOV Process } & \\
\hline & \multicolumn{4}{|c|}{ Multimedia Testbench } & \\
\hline \multicolumn{6}{|c|}{ Outcome Analysis } \\
\hline \multicolumn{2}{|c|}{$\begin{array}{c}\text { Blocking } \\
\text { Performance }\end{array}$} & $\begin{array}{c}\text { Bit Error } \\
\text { Rate }\end{array}$ & OSNR & \multicolumn{2}{|c|}{$\begin{array}{c}\text { Computation } \\
\text { Time }\end{array}$} \\
\hline
\end{tabular}

Figure 1. Proposed Study Architecture

\section{RESEARCH METHODOLOGY}

The proposed study of ORPT (Figure 1) is based on the potential multiplexing capabilities of WDM as well as routing based on wavelengths in mitigating the peak traffic issues in optical network. For the purpose of designing a better communication system considering the constraint of modelling the dynamic user behaviour, the lightpath that carries a single wavelength has to be established between the transmitting as well as receiving node in optical network. The proposed study consist of two wavelength assignment policy e.g. i) Arbitrary: selection of wavelength based on arbitrary selection of available wavelength and ii) Best Value: selection of best wavelength from the available wavelength for performing communication. The proposed system considers designing ROADM based on following principles:

1. Each add or drop ports of ROADM node should not be specific to any wavelength selection. The study considers that adding or dropping of any wavelength subjected at any ports of ROADM.

2. The add or drop port of any node should not be specific for any degree. The study considers that any channels to be added on a port can be actually directed to outgoing node degree and any channel to be dropped on a port can be directed to ingoing node degree.

3. Higher probability of cross connection within the node between node degrees (both incoming \& outgoing) and add or drop ports in ROADM.

The proposed ORPT considers designing a triple degree of ROADM node architecture that is subjected to color and directions at each add/drop ports. For this purpose, we select the design of arrayed waveguide grating as a multiplexer for fine tuning the capabilities of ROADM. The proposed ROADM module, exhibited in Figure 2, is basically highly enhanced version from our previous ROADM model in ECON [19]. The evolution of the proposed ROADM model is- Initially, the wavelength selective switching substitutes the arrayed waveguide grating on the drop port, while the couple component is substitutes the arrayed waveguide grating on the add port for supportability of $1^{\text {st }}$ principle of ROADM design. However, by doing this, the ROADM model doesn't guarantee to comply with $2^{\text {nd }}$ and $3^{\text {rd }}$ principle. In the final thoughts of design phase, the study chose to incorporate a number of add or drop ports that are universally shared by all the nodal degrees. 


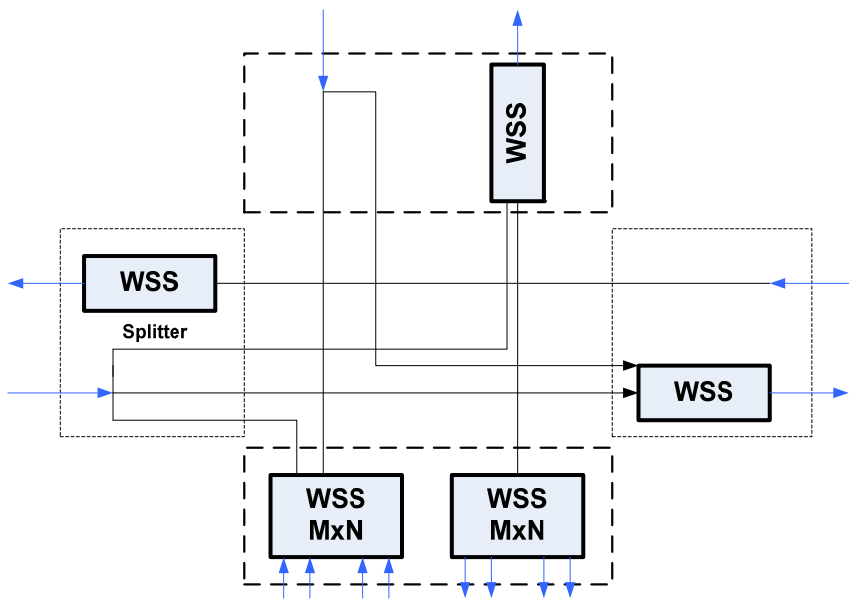

Figure 2. Proposed ROADM module

Figure 2 exhibits the enhanced ROADM model designed on the ECON framework [19]. The design principle of the above mentioned proposed ROADM module is incorporated with following charecteristics:

- The system will not consider any add/drop port for the ROADM model to be particular for any selection of wavelength. It can be applicable for all the general ports available in ROADM model.

- The system will not consider any particular degree for any add/drop ports of ROADM model. The study further ensures that added channels can be routed to the outgoing channels while dropped channels can be routed to the incoming channels.

- The system considers higher feasibility of the cross connection within the routed channels and node in the ROADM model.

The proposed ROADM model uses the Poisson inter-arrival concept for normalizing the incoming traffic that is mathematically represented as,

$$
l_{k}^{(i)}=\frac{\left(A_{i .} D_{m}\right)^{\lambda}}{\lambda !} l_{0}^{(i)}=\frac{\left(\frac{L_{i}^{\lambda}}{\lambda !}\right)}{\sum_{p=0}^{\eta} \frac{L_{i}^{p}}{p !}}
$$

The variables of eq(1) are likelihood $l_{\lambda}$ on the $\lambda$ wavelengths that are deployed on the $i^{\text {th }}$ link, the holding time $l_{\lambda}$ The system then considers the probability of distributed links of optical channel for allocated wavelengths at links $p$ and $q$ to be mutually independent that is mathematically represented as,

$$
P(\lambda)=\frac{\left(\begin{array}{c}
n_{p} \\
\lambda
\end{array}\right)\left(\begin{array}{l}
\eta-n_{p} \\
n_{q}-\lambda
\end{array}\right)}{\left(\begin{array}{l}
\eta \\
n_{q}
\end{array}\right)}
$$

The above eq.(2) considers $n_{p}$ and $n_{q}$ are the quantity of freely available wavelength on hop $p$ and $q$. $\eta$ is the amount of the wavelength in one optical fibre. The system then applies the provisional condition of probability for evaluating the distribution of busy wavelengths over the two hops channel as:

$$
m_{\lambda}^{(2)}=\sum_{i=0}^{\eta} \sum_{j=0}^{\eta} P(\eta-\lambda \mid \eta-i, \eta-j) l_{i}^{(1)} \cdot l_{j}^{(2)}
$$

The next phase of the study is focused on implementing our proposed queuing network model for optimizing both incoming and outgoing traffic using CTMP (Continuous Time MARKOV Process) [12]. Hence, 
applying the CTMP principle $(\mathrm{M} / \mathrm{M} / \mathrm{c})$, we explore the probability of idle wavelengths $\left(\lambda_{\text {idle }}\right)$ available on the $i^{\text {th }}$ hop as:

$$
m_{i}\left(\lambda_{\text {idle }}\right)=\frac{n(n-1) \ldots\left(n-\lambda_{\text {idle }}+1\right)}{\theta_{i}(1) \cdot \theta_{i}(2) \ldots \theta_{i}\left(\lambda_{\text {idle }}\right)} \cdot m_{i}(0)
$$

In the above eq.(4), the value of idle wavelengths $\left(\lambda_{\text {idle }}\right)$ is considered to be $1,2, \ldots . n$, where $\mathrm{n}$ is the highest number of the available wavelength in one hop $(n \epsilon \eta)$. Also, the probability value of $\mathrm{m}_{\mathrm{i}}(0)$ is computed as,

$$
m_{i}(0)=\left[1+\sum_{\lambda_{\text {idle }}=1}^{n} \frac{n(n-1) \ldots\left(n-\lambda_{\text {idle }}+1\right)}{\theta_{i}(1) \cdot \theta_{i}(2) \ldots \theta_{i}\left(\lambda_{\text {idle }}\right)}\right]^{-1}
$$

The proposed system therefore formulates an algorithm for checking the computation of blocking probability of the proposed system. The traffic model of the proposed system considers the simplex connectivity for establishing lightpath between the number of sources and destination based on the call arrival rate. The algorithm chooses to assign wavelength randomly after computing the busy and free hops in the available channel.

\section{RESULT ANALYSIS}

The outcome of the proposed system ORPT is compared with the Bathula et al. [21]. Bathula et al. [21] has attempted to enhance the performance of optical network from QoS viewpoint using OBS network for the purpose of minimizing the multi-constraint manycast issues in optical network. The study has formulated an algorithm mainly using shortest path tree to solve it considering BER, OSNR, and delay. The authors have studied the outcome using two performance parameters e.g. i) No. of Add/Drop Ports Degree and ii) BER. The outcome of the study is illustrated below:

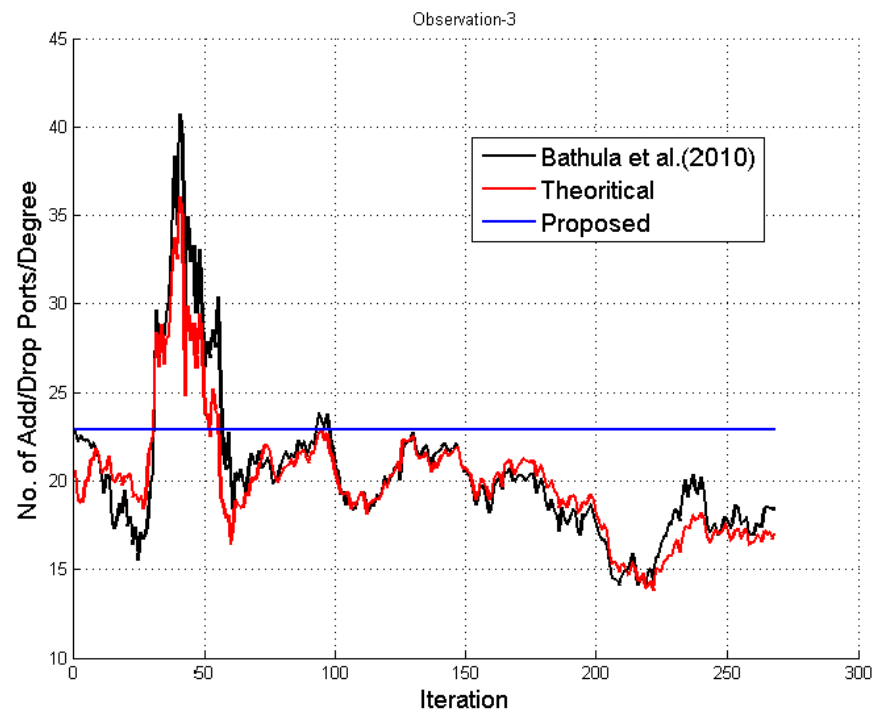

Figure 3. No. of Add/Drop Port Degree vs Iteration 


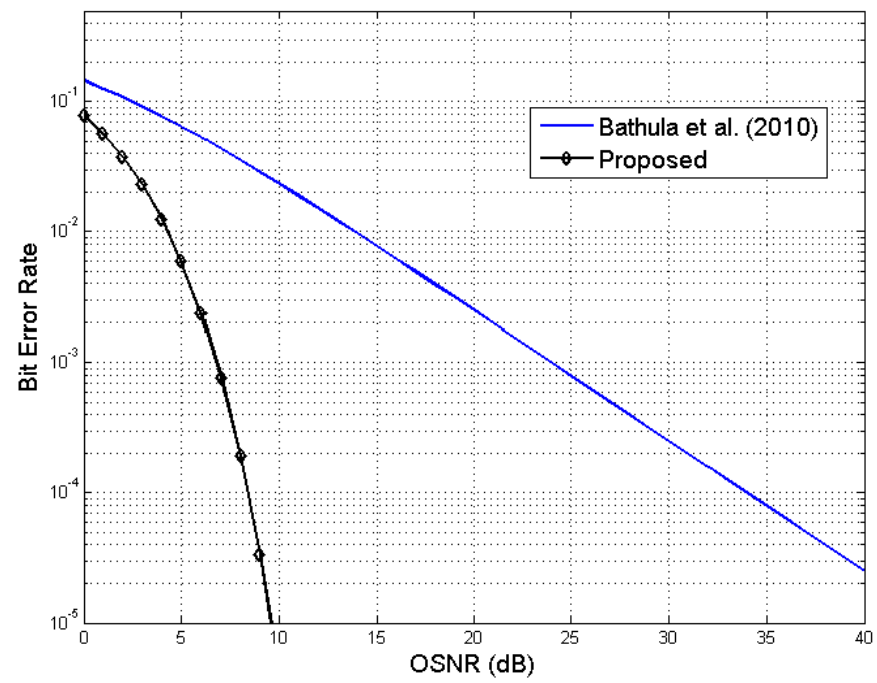

Figure 4. Analysis of Bit Error Rate Vs OSNR

Considering similar study parameters, the outcome is evaluated. The result exhibits the average blocking performance of both the system considering two service thresholds i.e. $\theta_{1}\left(\sigma_{\mathrm{th}}=4.25, \gamma_{\mathrm{th}}=0.9\right.$, $\left.\tau_{\mathrm{th}}=10 \mathrm{~ns}\right)$ and $\theta_{2}\left(\sigma_{\mathrm{th}}=4.25, \gamma_{\mathrm{th}}=0.8, \tau_{\mathrm{th}}=10 \mathrm{~ns}\right)$ respectively, where $\sigma_{\mathrm{th}}$ represents noise factors, $\gamma_{\mathrm{th}}$ represents reliability factor, and $\tau_{\text {th }}$ represents propagation delay. Retaining similar values for noise and delay factor, the outcome exhibited in Figure 2 and Figure 3 shows that with the decrease of the reliability factor from 0.9 to 0.8 have no impacts on the proposed system as compared to Bathula et al. [21] approach. In fact the proposed system has negative blocking probability till 0.15 loads in Erlang. The prime reason behind this outcome is that Bathula et al. [21] approach has adopted graph-based technique that doesn't effectively address the delay constraint of the burst. Hence, minimization of reliability constraint was found with increase in average call blocking. However, the proposed system introduced enhanced ROADM model using wavelength selective switching and can mitigate the delay as well as reliability constraint to a greater extent. Hence, optimal outcome of average request blocking probability is recorded for proposed study. Figure 4 exhibits the blocking performance for $7 / 4$ manycast configuration under similar service threshold $\theta_{1}\left(\sigma_{\mathrm{th}}=4.25, \gamma_{\mathrm{th}}=0.9\right.$, $\tau_{\mathrm{th}}=10 \mathrm{~ns}$ ). It can be seen that with the increase in the service request, there is also an increase in average request blocking on both the system. Considering the limits of probability, it was seen that Bathula et al. [21] approach was seen with exponential increase in blocking as compared to proposed ORPT scheme. The prime reason behind this outcome is that Bathula et al. [21] has attempted to minimize the QoS blocking using shortest path tree. Unfortunately, the technique gives rise to random contention on each optical path in case of any one of the destination node is unreachable. It also has adverse effect on the entire manycast request too. However, in the proposed system, it adopts OBS mechanism in different way 


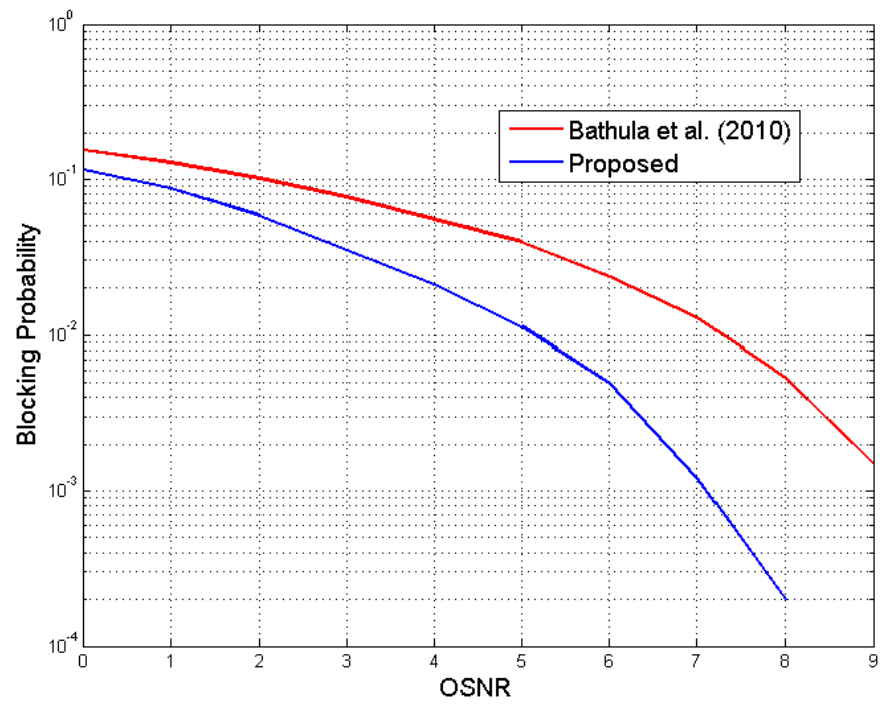

Figure 5. Analysis of Blocking Probability Vs OSNR

While Bathula et al. [21] have deterministically used OBS; proposed technique uses a concept of OBS on demand. The concept ensures that the sub-wavelength granularity is highly provisioned for allocating the signified wavelength into the burst. Hence, better processing of control packets as well as reserving resources for forwarding the burst and control message can be ensured. This is the sole reason of enhanced blocking performance of the proposed study. Hence, it can be said that proposed study introduces a cost-effective, simple, and robust routing performance that can model the peak traffic condition of optical network and ensure better data delivery among the nodes even in adverse traffic condition.

\section{CONCLUSION}

The proposed system is an extension of the ECON model that was discussed in our previous work. The ECON model was mainly a mathematical approach to enhance the OSNR whereas the proposed model ORPT discussed in this paper however incorporates a novel design of ROADM, WSS, as well as queuing network model to enhance the network performance in terms of routing behaviour in optical network. Tested with massive multimedia files, the proposed system is simulated under various environments to see that it performs optimal routing and have the capability of mitigating the uncertain traffic behaviour in optical network. It is known that optical networking is an upcoming topic of research and includes various hardware components to make it operational. Our study has attempted to introduce a computational method of optimizing the routing performance in peak traffic condition in optical network.

\section{REFERENCES}

[1] J.M. Simmons, “Optical Network Design and Planning”, Springer-Technology \& Engineering, 529 pages, 2014.

[2] C. Kachris, K. Bergman, I. Tomkos, “Optical Interconnects for Future Data Center Networks", Springer Science \& Business Media, Computers, 188 pages, 2012.

[3] P.V. Blanc, S. Figuerola, X. Chen, "Bringing Optical Networks to the Cloud: An Architecture for a Sustainable Future Internet”, Springer- uture Internet Assembly, LNCS 6656, pp. 307-320, 2011.

[4] J. Zyskind, A. Srivastava, "Optically Amplified WDM Networks: Principles and Practices", Academic Press, Technology \& Engineering - 512 pages, 2011.

[5] L.H. Chau, H. Hasegawa, K.Sato, "Hierarchical Optical Path Network Design Algorithm Considering Waveband Add/Drop Ratio Constraint", IEEE-Proceedings of COIN, 2008.

[6] G. Notamicola, G. Rizzelli, G. Maier, A. Pattavina, "Scalability Analysis of WSS-based ROADMs", IEEE-1 $7^{\text {th }}$ European Conference on Networks and Optical Communication, pp.1-6, 2012.

[7] S. Roy, A. Malik, A. Deore, "Evaluating Efficiency of Multi-Layer Switching in Future Optical Transport Networks", IEEE- Optical Fiber Communication Conference and Exposition and the National Fiber Optic Engineers Conference, pp.1-3, 2013.

[8] I. Karamitsos and C. Bowerman, "A Resource Reservation Protocol with Linear Traffic Prediction for OBS Networks", Hindawi Publishing Corporation Advances in Optical Technologies, Article ID 425372, 6 pages, 2013.

[9] D.A.B. Miller, "Reconfigurable add-drop multiplexer for spatial modes", Optics Express, Vol. 21, No. $17,2013$. 
[10] Y. Luo, N. Ansari, "A Computational Model for Estimating Blocking Probabilities of Multifiber WDM Optical Networks", IEEE communications letters, vol. 8, no. 1, January 2004.

[11] B.B. Gangaya, S. Talabathula, and P. Rajappa, "Estimating Blocking Probability based on Network Layer and Physical Layer Constraints for High Speed Optical Networks", Photonics 2006.

[12] X. Chu, T. Bu, XY Li, "A Study of Lightpath Rerouting Schemes in Wavelength-Routed WDM Networks", IEEEInternational Conference on Communication, pp.2400-2405, 2007.

[13] M. Bahleda, K. Blunar, I. Bridova, "The Blocking Probability Model in All-Optical Networks for Limited Wavelength Conversion", Journal of Computer Systems, Networks, and Communications, Article ID 289690, 10 pages, 2008.

[14] S. Ansari and A. Ansari, "Comparative Analysis of Routing and Wavelength Assignment Algorithms used in WDM Optical Networks", Research Journal of Applied Sciences, Engineering and Technology, vol.7, Iss.13, pp.2646-2654, 2014.

[15] J. Jeno Jasmine, J. Sutha, "Feedback Routing Algorithm in optical WDM Networks", International Journal of Computer Science \& Engineering Technology, Vol. 4 No. 10 Oct 2013.

[16] B. Zhang, J. Zheng, H. T. Mouftah, "Fast Routing Algorithms for Lightpath Establishment in Wavelength-Routed Optical Networks", Journal of Lightwave Technology, vol. 26, no. 13, 2008.

[17] K. Lu, G. Xiao, I. Chlamtac, "Analysis of Blocking Probability for Distributed LightpathEstablishment in WDM Optical Networks", IEEE/ACM Transactions on Networking, vol. 13, no. 1, February 2005.

[18] G.R. Kavitha, T.S. Indumathi, "Review of Challenges and Standard Research Contribution in Optical Networks", International Journal of Scientific and Research Publications, Vol.4, Iss.1, January 2014.

[19] G.R. Kavitha, T.S. Indumathi, "Enhanced Constraint-based Optical Network for Improving OSNR using ROADM", International Journal of Application or Innovation in Engineering \& Management, Vol.3, Iss.3, March 2014.

[20] Y. Pan, T. Alpcan, L. Pavel, "A System Performance Approach to OSNR Optimization in Optical Networks", IEEE Transactions on Communications, Vol. 58(4), pp. 1193-1200, 2010.

[21] B.G. Bathula, V.M. Vokkarane, "QoS-Based Manycasting Over Optical Burst-Switched (OBS) Networks", IEEE/ACM Transactions on Networking, vol. 18, no. 1, 2010.

\section{BIOGRAPHIES OF AUTHORS}

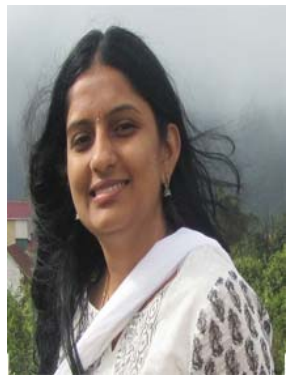

Kavitha G.R. has received Bachelor of Engineering in Electronics and communication and Master of Technology in Optical Communication Networking from Visvesvaraya Technical University, India.

She is currently pursuing Ph.D degree in Telecommunication Engineering from Dr. Ambedkar Institute of Technology affiliated to Visvesvaraya Technological University, India.

Her research Interest includes performanance analysis of ROADM optical Networks, Performanance analysis of WSS and OBS.

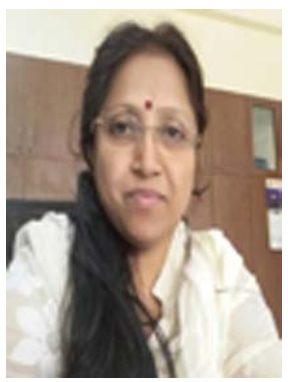

Dr. Indumathi T.S. has received B.E \&M.E from Bangalore University and Ph.D. degree from Visvesvaraya Technological University, India in 2011.

She is currently working as Professor and P.G. Coordinator, Bangalore region, Visvesvaraya Technicnological University, Muddenahalli, Chickaballapur District, Karnataka, India. She has 23 years of Teaching Experience and 10 years of Research Experience in the field of optical Engineering and Communication Networks.

Her primary areas of research are Optical Fiber Communication, System and Reconfigurability in Optical Networks. 